\title{
Técnica para el Estudio y la Investigación
}

\author{
por Lino Rodríguez-Arias Bustamante (")
}

SUMARIO: I-Creemos estímulos para la lectura. II-Aprendamos a estudiar. In-Algunas consideraciones sobre la investigación: $1^{\circ}$ Elección del tema. $2^{\alpha}$ Acopio de materiales. $3^{\alpha}$ Plan de trabajo. $4^{\alpha}$ Tarjetario bibliográfico. $5^{\natural}$ La redacción del trabajo de investigación. IV-Palabras finales.

I. - Creemos estímulos para la lectura.

Una de las deficiencias de la enseñanza de hoy día es que el estudiante alcanza sus estudios superiores, en bastantes ocasiones, sin saber a ciencia cierta cómo verdaderamente se debe estudiar. Podríamos decir inclusive, que existe una negligencia por parte del Estado en lo que se refiere a este aspecto de la educación.

En la época en que vivimos, donde el individuo se ve atraído $\alpha$ todas horas por toda clase de espectáculos y diversiones, hasta el extremo que su mismo hogar se ha visto invadido por los nuevos avances de la técnica, como la radio, los modernos tocadiscos y la televisión, muy poco se hace por contrarrestarlos eficazmente con el propósito de orientar a las personas hacia el libro. Por eso urge una mayor preocupación por dotar, organizar y fundar Bibliotecas en el país, y adoptar las medidas oportunas a fin de que la familia panameña se interese por frecuentarlas y beneficiarse de ellas; pues es triste comprobar hoy que ni las Bibliotecas de los grandes organismos se ven medianamente concurridas, lo que prueba a las claras que ni los profesores, ni los profesionales, ni los estudiantes universitarios sienten mayor interés por la lectura y el estudio.

Entendemos que sería muy conveniente que tanto las entidades públicas como las privadas destinasen un renglón de sus presupuestos a estimular la dedicación a la lectura y al estudio, estableciendo toda clase de certómenes $y$ concursos que invitaran a los individuos, desde su temprana edad hasta su madurez, a seguir los cauces de la vocación literaria o científica, que tan necesario es cultivar en los pueblos si se aspira a que tengan un alto nivel cívico y cultural.

(1) Catedrático de la Facultad de Derecho de la Universidad de Panamá.

Este artículo publicado en el Anuario de Derecho de Panamá. Vol VI, se reproduce con autorización de su autor. 
Empero difícilmente podrá uno acercarse al libro para estudiar si previamente no se ha creado el hábito de la lectura. Hay que aprender, primero, a saborear el libro, a comprenderlo y valorarlo en toda su amplitud. para después pasar a la faena mós dura del estudio y de la investigación.

Es menester que desde la infancia se dirija al niño hacia el libro. Que aprenda a amarlo entrañablemente, como al mejor de sus amigos. Por eso sus padres deben acostumbrarlo, desde pequeño, a frecuentar las librerías y a familiarizorse con las imágenes de los libros, para que en cuanto comience $\alpha$ asistir a la escuela ayudarle $\alpha$ crear su propia biblioteca.

El mayor galardón de un individuo debiera ser contar siempre con una buena biblioteca particular, seleccionada de acuerdo a sus aficiones $y$ profesión, para lo cual los Municipios sería conveniente contribuyesen con premios a las mejor organizadas.

No se pierda de vista que vivimos en unos tiempos en que priman los bienes materiales sobre los culturales, motivo por el cual hay que desplegar todos los esfuerzos posibles para sanear este ambiente refractorio al libro y proporcionar los instrumentos que vayan erosionando las prácticas malsanas en la comunidad, con el objeto de que los individuos vuelvan $\alpha$ sentirse - como en antaño- atraídos por la soledad y el recogimiento del gabinete de estudio.

Sabemos que esto resulta más árduo en climas tropicales, donde todo invita a ser extravertidos: el buen tiempo, el calor, los ruidos,... Sin embargo, fundemos cooperativas de libros, tertulias, revistas, periódicos... todo lo que contribuye a enriquecer la cultura patria hasta conseguir entusiasmar a la gente: a que cultive los bienes del espíritu.

Después que se haya creado el hábito de la lectura tendremos hombres cultos que no disiparán sus ratos de ocio y serán más disciplinados y responsables en el ejercicio de sus oficios y profesiones.

Tan solo así la educación empezará a enrumbarse rectamente en la vida nacional y contaremos con jóvenes dedicados con fruición a sus estudios; generaciones que en el día de mañana tendrán la suficiente preparación para ocupar los cargos de responsabilidad en la empresa privada y en la Administración del Estado.

\section{II. - Aprendamos a estudiar.}

Cuando se tiene el hábito de la lectura es más factible crearse el del estudio. Obvio es que no siempre es así, pues a veces el individuo se ha entregado al libro fácil, de entretenimiento o nocivo, sintiéndose incapaz de abordar el estudio disciplinado y metódico. Sin embargo, la persona acostumbrada a la lectura se encuentra, por lo general, en condiciones favorables para iniciarse en el estudio, pues es lo natural que posea un vocabulario que la facilita enfrentarse con la comprensión del texto que deba aprehenderse. Además ha adquirido un adiestramiento en el manejo de los libros, que le habró servido para dar agilidad a su mente e imprimir vuelos a su imaginación, lo cual es necesario tanto para la información como para la formación humana.

Es natural que tropiece con más dificultades en sus estudios aquel individuo que ha seguido las enseñanzas primaria y secundaria de forma 
deficiente y se encuentra al llegar a los cursos superiores sin hábito de estudio y con una pobreza en su vocabulario. Este mal estudiante y pésimo lector tiene ante sí un porvenir poco halagüeño a no ser que reaccione con tesón ante la nueva etapa de su vida que se le presenta en la Universidad.

Entonces el estudiante lo primero que tiene que hacer es tratar de crearse un hábito de estudio, para lo cual procurará diariamente dedicarle cierto tiempo a sus tareas universitarias. Esto habrá de complementarlo con la asistencia a clase con asiduidad procurando asimilar los conocimientos fundamentales de la asignatura y aclarar aquellos conceptos que se le presentasen oscuros. A esta labor le ayudará si, previamente a la asistencia a la cátedra, repasa el tema que vaya a explicar el profesor y toma algunas notas sobre lo que él ha comprendido de las varias lecturas que le haya dado.

Es aconsejable que el alumno siempre asista a clase cón un cuaderno de notas en el que apunte lo sobresaliente de la exposición de su maestro. Recuerdo que, durante mis estudios universitarios, siempre me ponía de acuerdo con un compañero de aula con el objeto de recoger las explicaciones entrambos $y$ después confrontarlas con varios libros sobre la materia. Ahora algunos universitarios se valen de la grabadora que registra la intervención del catedrático y luego pueden escucharla en casa cuantas veces quieran. De todos modos resulta muy importante que el estudiante de la cátedra no se limite a ser un sujeto pasivo que se aguanta pacientemente la conferencia del profesor sin importarle mucho lo que éste expresa. Por el contrario, debe adoptar la actitud de un sujeto activo, dinámico. operante, que toma apuntes e interroga al maestro para aclarar conceptos $y$ todo ello lo conserva cuidadosamente para examinarlo detenidamente en la quietud de la biblioteca o en su hogar.

Actualmente se impone la cátedra comunitaria, en el sentido de que el profesor no debe limitarse a exponer una lección magistral sino que está obligado a hacerse comprender de los estudiantes, estimulándolos $\alpha$ que intervengan preguntando, si bien evitará que sus intervenciones se aparten de la cuestión tratada, pues en este caso se rompería la disciplina académica y se perdería la clase en meras divagaciones nada constructivas ni provechosas.

A este propósito conviene responsabilizar a los estudiantes en la preparación de temas que versen sobre la asignatura explicada a fin de que los desarrolien por escrito o en intervenciones orales ante sus compañeros, bajo la dirección del catedrático, seguidas de un lapso de preguntas por los asistentes a la clase.

Es decir, hay que insistir en que el estudiante se decida a forjarse personalidad, que no sea pues un simple emulador de su profesor. Porque en cuanto más conciencia tome de sí mismo, más respetará y admirará la sapiencia y las condiciones personales de su maestro. Esta integración reciproca entre profesores y estudiantes consigue dar mayor realce a la cátedra, por cuanto demuestra que el alumno desde el comienzo es considerado como un ser pensante capaz de asimilar las enseñanzas del maestro y. a través de ellas, formarse su propio criterio.

La participación oral del alumno en la clase debe versar -como decimos- sobre uno de los temas que aparecen en el programa de la asig- 
natura. El estudiante no se limitará a repetir el pensamiento del profesor expresado en los apuntes o en el libro, sino que habrá de ampliarlo con la consulta de otros autores.

Su intervención estará dividida en tres partes: "en la primera o introductoria, expondrá el plan de su trabajo y la bibliografía utilizada; en la segunda, se desarrollará la cuestión medular de la tesis a base de exponer el concepto, su formación histórica, la referencia al Derecho comparado, su naturaleza, las teorías sobre el tema, las relaciones con otras instituciones y clasificaciones; $y$, en la tercera, se haró referencia $\alpha$ las conclusiones, donde el expositor resumirá sus puntos de vista y las lagunas y reformas que según él debiera introducirse en la materia abordada.

A la cátedra el estudiante llevará un guión o esquema que le serviná de orientación en su exposición oral; pues debe acostumbrársele a hablar ante sus compañeros empleando un lenguaje académico que le facilite fxmiliarizarse con los términos técnicos de la disciplina que estudia. Cuidará la forma en la intervención, de manera que no repita indebidamente palxbras o utilice giros mal construídos, porque ya nos decía IHERING que la ley de la belleza ha de tenerse siempre en cuenta en las construcciones juŕdicas. Esa elegancia que tan a gala tuvieron los juristas romanos, tanto en sus escritos como en su oratoria, y que fué una de las razones de su grandeza. Por eso al estudiante hay que enseñarle a que se exprese con clanidad lo mismo cuando habla que cuando escribe, $y$, por lo tanto, si al estudiar tropieza con pasajes oscuros - claro es, que dentro del nivel técnico en que se desenvuelve - es preferible que los pase por alto y trate de com. prender el resto del discurso, porque a lo mejor éste, estudiado en su totalidad, le ayudará a comprenderlos más tarde.

De aqui lo conveniente de que el estudiante cuando estudie lea primero el tema una vez o varias y después trate de asimilarlo párato por párrafo apuntando en una libreta los conceptos más fundamentales. Después los repasará tratando de memorizar lo esencia! de la materia, lo que le permitiró ordenarla adecuadamente en su intelecto. Sin embargo, no debe abusar de la memoria, pues antes de registrar las ideas en ésta tendrá que haber comprendido bien el discurso. Intentar memorizar los conceptos sin comprenderlos es tanto como exponerse a correr un grave riesgo, porque se convertirá en un recitador de palabras sin alma y sin sentido. Por este camino será un papagayo que habla y habla y nunca sabe lo que dice. Jamás tomará conciencia ni de las cosas, ni de los hechos, ni de las ideas.

Nosotros, por el contrario, creemos que del estudiante hay cue hacer sobre todo un hombre de vasta cultura y de sólida formación moral, a cuyo fin es menester que no se limite a estudiar los apuntes o el libro que señale su profesor, sino tratar de ampliar sus conocimientos consultando la bibliografía clásica y moderna.

Un estudiante que sabe catar la lectura y el estudio es una promesa para la investigación.

\section{III.- Algunas consideraciones sobre la investigación.}

Es una cuestión sumamente grave el que los estudiantes pasen su: tiempo en la Universidad $y$ no aprendan a investigar, lo cual prueba que 
el centro universitario no es capaz de cumplir uno de sus fundamentales cometidos; pues, como ha recogido José SANCHEZ FONTANS, la idea esencial de la Universidad es que ésta sea una "comunidad de profesores y estudiantes para la investigacićn y difusión de la verdad". (1)

$A$ este objeto, el estudiante ha de someterse a una metodología que le conduzca racionalmente al conacimiento integral de la materia que investiga, para lo cual habrá de dividir su estudio en varias etapas:

\section{la. Elección del tema.}

Es al alumno a quien corresponde elegir el tema de su investigación, de acuerdo con sus conocimientos y preferencias, en lo cual a veces habrá influído casi decisivamente la explicación de los profesores; pues hay quienes hacen amena la materia árida, mientras que otros convierten en odiosas o poco atractivas asignaturas a que el estudiante se siente, por lo generol, atraído. De todas maneras al estudiante es a quien atane buscar el tema de su investigación, siendo fiel a su vocación, a cuyo objeto, desde que se inicie en sus estudios universitarios, deberá tratar de ampliar los conocimientos que recibe en la cátedra, conforme a sus inclinaciones. Es muy conveniente que, desde el primer momento, se asesore de especialistas capaces de orientarlo por los ccuces de la asignatura o de las asignaturas de su predilección, porque así se economizará investigaciones infructuosas y podrá, desde el comienzo, lograr una buena selección de su bibliografía.

Obvio es que este asesoramiento profesoral que tanto recomendamos dejaría de cumplir su loable cometido, cuando el estudiante pretendiese llevarlo al extremo de recostarse demasiado sobre él no dando un paso en su investigación $\sin$ el consejo del catedrático. Está bien que así sea, en lo fundamental; pero nunca que suponga matar la iniciativa del investigador y. menos aún, hacerle excesivamente cómoda su labor. El proceso de la investigación equivale al parto de la mujer con dolor. El que no lo entiende de esa forma es mejor que ni siquiera lo acometa. Los resultados del esfuerzo intelectual se miden por la capacidad de sacrificio del investigador.

Ahora bien; el estudiante no puede hacer imposibles en la investigación. Por eso sus esfuerzos estarán condicionados a la biliografía de que pueda disponer y a sus conocimientos de idiomas. Esio es lo que nos lleva a insistir en que es menester organizar bien nuestras bibliotecas y estimular en los alumnos el hábito de la compra de libros. Igualmente hay que enseñarles el manejo de otras lenguas además de la propia. Hoy día es casi inverosímil investigar sin el manejo de varios idiomas; depende de la clase de investigación. Difícilmente se conseguirán excelentes estudios en el campo del Derecho romano sin el conocimiento del latín; por lo general, en el ámbito del Derecho se necesita, por lo menos, el dominio del francés y el italiano. Los juristas italianos han tenido la ventaja de que han sabido asimilar el pensamiento jurídico alemán a su mentalidad latina lo cual facilita enormemente el estudio de la ciencia jurídica italiana, que se ha enriquecido con la técnica germana sin perder por ello su fisonomía propia:

1.- "Consideraciones metodológicas sobre los seminarios", Montevideo, 1962, p. 11. 
Entre nosotros, en la América Latina, se descuida excesivamente la formación idiomática del estudiantado, lo cual es muy lamentable porque nos coloca en una situación de inferioridad para realizar labor de investigación, contribuyéndose así a perpetuar un "coloniaje intelectual" de consecuencias funestas en el campo del desarrollo y progreso de nuestras nacionalidades.

\section{2a. Acopio de materiales.}

Ya decidido el estudiante por un tema, se inicia una segunda etapa que consiste en tratar de familiarizarse con él, antes de comenzar a elaborar las fichas o tarjetas. Pues sería difícil ponerse a hacer tarjetas bibliográficas, sin previamente haberse formado una idea general de los problemas concernientes al tema por medio de la lectura de varios trabajos fundamentales sobre él. De otra manera se incurriría en el error de tomar notas que se considerarian de importancia $y$. sin embargo, no lo serían; 0 , por el contrario, se dejarían de tomar notas de cosas importantes.

Inmediatamente debe realizarse el acopio de material, que es el fundamento de toda investigación. Es menester que se reúna el mayor número de bibliografía relacionada directa o indirectamente con la materia objeto de la investigación. Debe seguirse el orden de recoger primero notas de aquellos libros que de una manera general se ocupan del tema, cuyos tratadistas han alcanzado cierta autoridad en el campo que se investiga (filosófico, civil, penal,...); es decir, aquelias obras que, como nos dice Carlos BOSCH GARCIA, tratando de la materia general, incluyan en su capitulo o en párrafos sueltos conceptos relativos al tema escogido por el autor, que le facilitarán situarle en un panorama general de conocimientos mucho más amplios. (2)

La realización de esta primera empresa por parte del investigador exige llevar a cabo una selección de los mós autorizados libros sobre la materia, los cuales no es necesario que lea en su totalidad, sino que únicamente indague, de acuerdo con sus índices, en los puntos que se refieren al tema elegido, insistiendo sobre todo en captar el pensamiento de los cutores consultados.

Después se tratará de seleccionar el material bibliográfico que aparezca en las Revistas. A veces esta investigación se descuida, siendo ello una falla imperdonable; pues, por lo general, los artículos aparecidos en Revistas recogen lo más sustancioso de las últimas posiciones doctrinales. De aquí que los buenos libros siempre sean el fruto de los escritos que previamente se publicaron en las Revistas. Para su localización pueden orientar los mismos tratados generales que se hubieran consultado, como asimismo los índices de Revistas que publican las mismas editoriales $u$ otras pu. blicaciones y las secciones de Revista de Revistas.

Por último, o simultáneamente, habrán de consultarse los estudios monográficos escritos sobre el tema. Esta tarea hay que hacerla de forma minuciosa: pero sin dejarse impresionar demasiado por una especial mono-

2.-_'La técnica de investigación documental”, México, 1963, p. 13. 
grafía que pudiera restar originalidad a la obra fruto de la investigación. Indiscutiblemente, que en casi todos los temas existen algunos libros monográficos o artículos de Revistas que son imprescindibles para realizar el cometido propuesto, hasta el extremo que sin la posibilidad de su consulta hay que abandonar la investigación. Es de todo punto inútil intentar hacer nuevas aportaciones en un campo dado de la investigación, cuando se desconocen algunos de sus más fundamentales jalones. Por ello, si a la vista del tema objeto de investigación se descubriese la existencia de monografías que por su reciente publicación y por su madurez científica se hace difícil superarlas o aportar nada nuevo. lo más prudente es que se cambie de tema, desde el instante que la investigación sería totalmente infructuosa.

\section{3a. Plan del trabajo.}

Nos dice J. LASSO DE LA VEGA, que conocido el tema, averiguado lo que se ha escrito sobre él y formada la bibliografía, procede trazar el plan. El plan viene a ser como la osamenta del trabajo, el esqueleto, la estructura sobre que ha de levantarse el edificio". (3) O sea, que con los anteriores elementos de juicio el estudiante se encuentra ya en disposición de elaborar un esquema mental de su futuro trabajo: pues de lo contrario se expondrá a leer y a tomar notas sin orden ni concierto que luego a la hora de la confección del trabajo resultaría que no le serían de gran utilidad e inclusive podrían faltarle otras consultas de carácter necesario. De ahí la importancia de los ajustes desde el comienzo del trabajo. Hay que establecer, lo más pronto posible, un método, un orden lógico lo más rigu10so. Es como quijen se va a hacer un traje a la medida. El sastre no puede ponerse $\alpha$ cortar paño aquí y allá sin un patrón. Tiene que trazar tam. bién su esquema o plan. Para ello toma las medidas de su cliente con el objeto de que luego el traje confeccionado se ajuste bien a su cuerpo. Y así, posteriormente, se producirán las pruebas del proyectado traje; y el sastre con su tiza y su cuaderno de notas apuntará las enmiendas que haya de introducir con el fin de que su obra sea lo más perfecta.

Le ocurre lo mismo al investigador. Al iniciar su labor, una vez acopiado el material, tiene que entregarse a elaborar un plan, que no es definitivo ni mucho menos. El ha de sufrir todas las reelaboraciones necesarias antes de la redacción final de su trabajo.

Este proyecto de plan contendrá capítulos, divisiones y subdivisiones, a fin de que se delimite con rigor metódico el campo de las futuras investigaciones. Así LASSO DE LA VEGA nos dice, que "si en los campos del fútbol no estuvieran pintadas sobre el suelo las líneas que delimitan el área de juego, los jugadores se saldrían continuamente de ella; así, si el investigador no comienza su trabajo por trazar los límites de su tema, correrá riesgo constante de elaborar capítulos que más tarde comprobaría que se salen fuera del área escogida". (4) Por lo tanto, cuanto con mayor precisión el investigador ajuste su proyectado plan será más el fruto que obten-

3.--“"Cómo se hace una tesis doctoral". Sen Sebastión, Ed. Internacional, 1947, p. 159. 4.-Op. cit., p. 160 . 
ga de su esfuerzo intelectual, desde el momento que al ceñirse a un orden lógico consigue evitar incursiones en campos no relacionados con su trabajo. Esto no quiere décir, que en la medida que le sea posible trate de enfocar las cuestiones de su investigación dentro del más vasto nivel cultural a cuyo objeto procurará documentarse bien sobre todas aquellas materias que directa o indirectamente se refieran a su tema.

\section{4a. Tarjetario bibliográfico.}

Una vez que el estudiante se ha formado una idea general del tema sobre que va a investigar, a base de la lectura de los libros fundamentales. tiene que dedicarse inmediatamente a tomar notas de lo que lea, pues de otra forma perdería lamentablemente su tiempo: $y$, cuando uno prepara un trabajo de este tipo, apremia aprovechar hasta el máximo la dedicación procurándose que todo quede apuntado y debidamente organizado, puesto que habrá de consultarse una pluralidad considerable de libros, artículos, mónografías, documentos y jurisprudencia.

Un procedimiento rudimentario, desechado ya por ser poco práctico, consiste en escribir en cuadernos las notas que se toman de las obras que se consultan; pero este sistema presenta el inconveniente de lo difícil que se hace el aprovechamiento de lo anotado, desde el momento que, por tratarse de hojas fijas, es muy arduo conciliar las citas recogidas sobre el mismo concepto, que por ser vertidas por diferentes autores, su ordenación adecuada requeriría hacer un indice de dichos cuadernos de notas, lo cual sería sumamente complicado.

También se utiliza el método -si la investigación es más bien a largo plazo de que cuando se lee un libro se hace de él un índice de materias de acuerdo a la apreciación subjetiva del lector; pues de esta manera lo tendrá siempre listo para la consulta: claro es que partiendo de la base de que forme parte de su bibliateca particular. Este sistema es muy apropiado con libros que le pertenezcan a uno, pues sin necesidad de molestarse en ficharlos los podrá manejar fácilmente a través del índice de materias que reenviará a los conceptos desarrollados por el autor de su. obra.

Sin embargo, el sistema moderno de mayor éxito es el del tarjetario bibliográfico. Para su confección es preciso que el estudiante se disponga a realizar un doble trabajo: por un lado, a anotar en tarjetas movibles de 3" $\times 5^{\prime \prime}$ (pulgadas) el título de cuanto libro, monografía, artículo, etc.. tenga noticia que verse sobre su tema y que irá ordenando en un fichero apropiado para ellas conforme al plan provisorio que se trazara: $y$, de otra parte, tomará nota de las publicaciones consultadas - sujetándose a los conceptos de su plan provisional- en tarjetas de $4^{\prime \prime} \times 6^{\prime \prime}$ (pulgadas) que se alinearán de acuerdo a las clasificaciones, capítulos y epígrafes en que él hava dividido su tema. Cuidará de anotar, sobre todo en la tarjeta bibliográfica, el nombre de la biblioteca en que se encuentre la obra fichada, para que asi le sea fácil localizarla cuando le llegue la hora en que tenga que consultarla. 
La tarjeta bibliográfica es preferible que sea del tamaño que señalamos más arriba, porque de ser más grande se clesperdiciaría mucho espacio, dado que debe confeccionarse de la manera siguiente:

GUTIERREZ, Carlos José

Lecciones de Filosolia del Derecho

Madrid, Ed. Tridente, 1963

Es decir, que dicha tarjeta llevará anotado lo imprescindible para localizar en cualquier momento el libro. $\mathrm{Si}$ no está en la biblioteca particular deberá indicarse en la que se halle.

Así, pues, se pone, en primer lugar, el apellido del cutor en mayúscula cerrada y a continuación su nombre en minúscula, debajo el título del libro subrayado y, finalmente, la población en que se editó. la editorial, el año, la edición si existe más de una, el tomo, el volumen, cuando haya varios, y el número total de página de la obra. De esta manera se verá que será dificilísimo que con todos esos dátos no se pudiera encontrar el libro en la biblioteca, si ésta se halla medianamente organizada.

En el supuesto de que se trate de un crtículo de Revista la fiche se anotará también en una tarjeta de $3^{\prime \prime} \times 5^{\prime \prime}$ (pulgadas), como el modolo siguiente:

\section{OLARTE, Teodoro}

"Panorama de la filosofía hispanoamericana del siglo XX"

Revista de Filosofía, Universidad de Costa Rica, 1958, Núm. 3, pp. 207 s.s.

O sea, que se escribe en primer término el apellido del autor en mayúscula cerrada, seguidamente el nombre en minúscula, debajo el título del trabajo entre comillas, después el título de la Revista subrayado, el lugar de su publicación, año, número, o una u otra cosa, y la página en que comienza el trabajo o entre las páginas en que se haya comprendido; v. gr., pp. O págs. 207-220.

Estas tarjetas bibliográficas se agruparán en un fichero de análogas dimensiones conforme al plan que esbozara el investigador o de acuerdo con un sistema de conceptos que él haya establecido. Este último criterio es seguido cuando se organiza la investigación con un carácter permanente con la finalidad de hallarse al día en determinadas malerias científicas. Dentro de cada apartado o concepto se ordenarán las tarjetas siguiendo la clasificación alfabética del apellido de los autores. También se puede adoptar un orden cronológico de acuerdo a cuándo haya sido registrado el libro 0 artículo de Revista en la biblioteca. En este caso, generalmente el libro lleva una referencia a fin de poderlo localizar, independientemente de los datos que toda ticha bibliográfica tiene; por ejemplo, LJ-3, que se escri- 
be en la parte superior a la derecha de la tarjeta, y significa que dicho libro estó clasificado en la sección de Lógica Jurídica con el número 3. Así.

GARCIA MAYNEZ, Eduardo

LJ-3

Lógica del Juicio Jurídico

México, Fondo Cultura Económica, 1955.

O también:

PRECIADO HERNANDEZ, Rafael

FD-3

Lecciones de Filosofía del Derecho

México, Ed. Jus, 2a. ed.، 1954.

Esto significa, que el libro está clasificado en la sección de Filosofía del Derecho con el número 3.

Puede ocurrir que la obra de un autor ya estuviera clasificada en la biblioteca $y$, posteriormente, se publique una nueva edición $u$ otro libro del mismo autor sobre la misma materia, en cuyo caso se puede resolver el problema de esta manera:

FD-1

RECASENS SICHES, Luis

Vida humana, sociedad y derecho

México, Ed. Porrúa, 3a. ed., 1952

FD-1A

RECASENS SICHEZ, Luis

Tratado general de Filosofía del Derecho

México, Ed. Porrúa, 1959.

O también:

FD-2

LEGAZ LACAMBRA, Luis

Filosotía del Derecho

Barcelona, Ed. Bosch, 1951.

FD-2'1

LEGAZ LACAMBRA, Luis

Filosofía del Derecho

Barcelona, Ed. Bosch, 1961.

Ásí, de este modo, se consigue que estén juntas las tarjetas que versan sobre el mismo tema, aún cuando sean, de autores distintos, v. gr.: 
RUSZKOWSKI, Āndrés

El comunismo

Lima, 1961 .

CRONYN, George W.

200 preguntas $\mathrm{Y}$ respuestas sobre el comunismo

México, 1962.

Es decir, que estas dos obras se encuentran clasificadas, dentro de un criterio amplio, en la sección de Sociología, y el investigador puede consultar de una vez todas las que se refieren al mismo tema.

Otras veces puede suceder que la tarjeta bibliogrática aparezca en un apartado $\mathrm{y}$ el libro, por tratar también de distintas materias, esté clasificado bajo concepto diferente, en cuyo supuesto hay que hacer una referencia; v. gr.:

SCIACCA, Michele Federico

La struttura della libertá nella Costitutione ontologica del'uomo

en Comunicaciones Introductorias (Ho-23)

Esto es: esta tarjeta se halla clasificada bajo el concepto de "Libertad": pero, sin embargo, la obra se encuentra en el apartado "Hombre", con el número 23, puesto que también se ocupa de esta materia.

El estudiante habrá de iniciar su investigación dirigiendo su acción a las cuestiones siguientes: la. A la lectura de algunos libros fundamentales para formarse una idea sobre el tema en el supuesto de que careciese de ella. 2a. A esbozar el plan de su trabajo, que deberá reelaborar cuantas veces crea necesario hasta conseguir su plan definitivo. 3a. A preparar su tarjetario bibliográfico. 4a. A recoger cuanta idea original se le ocurra sobre su tema. Y $5 a$. A organizar su tarjetario bibliográfico del contenido de las obras consultadas.

Llegará un momento en que estas cinco cuestiones estarán en marcha simultáneamente, pues la consulta de las tarjetas bibliográficas de acuerdo al plan del tema, servirá para organizar el fichero de tarjetas de contenido, y la lectura de las obras, sugerirán tanto ideas personales al cutor como la introducción de modificaciones a su plan originario.

$Y$ así llegamos al tarjetario del contenido de las obras consultadas, que nos van a proporcionar el fondo de la investigación, así como las tarjetas bibliográficas y el plan constituyen el aspecto formal del trabajo.

Como decíamos, una vez que se pone en marcha el primer plan provisorio, hay que empezar a tomar notas de cuanta obra se consulte. Ya desechamos el procedimiento anacrónico de los cuadernos y nos pronunciamos a favor de las tarjetas movibles e indicamos es preferible que se utilicen tarjetas de $4^{\prime \prime} \times 6^{\prime \prime}$ (pulgadas). Preferimos éstas, debido a que si fueran de un tamaño mayor a veces habría que desperdiciar bastante espacio, puesto que en cada tarjeta se ha de recoger a ser posible un solo concepto como único medio de poder luego clasificar juntas aquellas que se refieren $\alpha$ un solo punto de la materia, aún cuando correspondan a distintos autores. Así, v. gr.: 


\section{Derechos humanos Igualdad}

Desde que se inventaron los Derechos del hombre todo imbécil los sabe de memoria para explotarlos, como si la igualded ante la ley implicara una equivalencia de aptitudes.

INGENIEROS, José, El hombre mediocre

Buenos Aires, Ed. Meridion, 1953, p. 161.

Luego, puede advertirse, que en la parte superior de la izquierda de la tarjeta se escribe el concepto genérico sobre que se trabaja y en la derecha, el específico. Después viene el texio donde se transcribe literaimente el pensamiento del autor que se glosa; $y$, finclmente, se reproduce la tarjeta bibliográfica, copiando el apellido en mayúscula cerrada y el nombre en minúscula del autor, el título de la obra subtayado. lugar de su publicación, editorial, año y página en que aparece en el libro la cita.

Hemos dicho que debe transcribirse literalmente el pensamiento del autor, pues de esta forma no habrá nunca duda sobre si se interpretó bien - mal lo que dijo, aparte de que hecho de esta guisa siempre existe posibilidad de que en el momento de redactar el trabajo de investigación se puede recoger su pensamiento literalmente o no, o parte en forma literal y otra tan solo tomando su idea. Por eso la tarjeta de contenido elaborada a base de hacer un resumen de los párrafos que interesan del libro consultado, no ofrece ninguna garantía; pues siempre quedará la duda si se captaron fielmente o no, obligándonos a volver de nuevo a la obra a fin de cerciorarnos de ello, lo cual hace ineficaz el trabajo anteriormente realizado. mientras que la transcripción literal de los párrafos de la öbra a la tarjeta, le da a ésta un valor permanente que evita tener que volver a consultarla.

Ahora, ha de tratarse que las citas no sean excesivamente largas, aún cuando las tarjetas se pueden escribir por ambas caras, porque de ser así la copia se extendería a varias páginas de la obra lo cual haría después difícil, cuando se escribiese el trabajo de investigación $y$ se tomase algún párralo de lo copiado, saber a qué página correspondería. De aquí que insistamos en la necesidad de hacer citas breves que recojan un solo concepto, pues de lo contrario después no se podría llevar a cabo la clasificación de las tarjetas conforme a los apartados y subapartados que figuren en el fichero, como transcripción del plan del trabajo o del sistema de conceptos elaborados por el investigador. Āsí, por ejemplo:

\section{Derecho}

\section{Coactividad}

En el orden conceptual hay que mantener la distinción y subrayar la coactividad como nota del Derecho. El no necesitar recurrir a la coacción será una prueba de perfección humana. Pero bien sabemos que, aún en esos sectores más elevados, la confianza y la genèrosidad y hasta el sentido del interés nuestro fallan con frecuencia, $y$ surge entonces la fuerza como recurso extremo al servicio de la paz y de la justicia. La coactividad, al cabo, responde a la caída original del hombre. 
CORTS GRAU, losé. Curso de Derecho Natural

Madrid, Ed. Nacional, 1953, p. 229.

Es necesario también, repetir en cada tarjeta la ficha bibliográfica completa del autor consultado, dado que estas tarjetas han de hallarse sometidas a constante clasificación y se da con trecuencia el caso de consultar varios libros o artículos de Revistas de la misma persona. Entonces resultaría que de no copiar en cada tarjeta la obra no se sabría que trabajo se estaría citando, aparte de que la cita incompleta de la obra constituye una molestia, desde el momento que obliga al investigador a buscar continuamente la referencia para poderla citar de manera adecuada.

Es admisible recurrir también a la cita indirecta, cuando no se puede encontrar la obra original, pero procurán ¿ose no abusar de ella y que sea de libros poco utilizados, desde el momento que desdeciría mucho hacer citas indirectas de libros frecuentemente usados. Así, v. gr.:

\section{Filosatia}

Poesía

Rousseau no sólo profesa en teoría la filosofía del sentimiento, como los moralistas ingleses de su tiempo, que son aún intelectualistas y analizadores que disertan sobre la sensibilidad. Muy a menudo se ha insistido en cómo y con qué intensidad él mismo es todo sentimiento: en cómo vive con todas las fibras de su ser y con una especie de heroísmo la primacía de la sensibilidad. De aquí que Baumier, en su libro Tumba y apoteosis de Rousseau, escribe: "cantemos, celebremos la presencia del nuevo Dios del sentimiento".

Tableau des moeurs de ce siécle on forme d'épitres, Londres $Y$ París, 1788 (Masson, 111, 76) cit. por MARITAIN, Jacques. Tres reformadores, Madrid, Ed. Epesa, 1948, p.p. 128 y 146.

La confección de este tarjetario de contenido puede hacerlo el investigador a medida que consulta las obras, lo cual es más lento, pero ayuda a fijor más los conceptos que se manejan; o también es posible acudir al procedimiento de señalar la parte a copiar en el libro metiendo tarjetas en blanco con los encabezamientos genérico y específico, escritos a lápiz para que se borren fácilmente, y después dórselo a la mecanógrafa para copiarlas.

Finalmente se procede a la clasificación de las tarjetas en un fichero adecuado conforme al plan del tema o al sistema de conceptos establecidos por el investigador, ordenándose de acuerdo a la secuencia lógica exigida por la materia tratada, para que el material allí recogido y en la forma en que aparece, sea ya el esqueleto del libro, de la monografía o. del artículo de Revista proyectado.

5a. La redacción del trabajo de investigación.

En este momento el estudiante se encuentra ante la etapa final de su investigación. 
Comienza el momento de la redacción. En una presentación o Introducción, escrito al principio o después de haber redactado el cuerpo del estudio, se explicará en forma clara, breve y amena los objetivos que se propone el autor en su obra. Como decimos, este capítulo no debe ser muy largo, ni detallista en su exposición. Recogerá, a grandes trazos, los puntos sobresalientes que se desarrollan a continuación, para entrar definitivamente $\alpha$ redactar su estudio.

Aquí viene el manejo del fichero de las tarjetas de contenido y las notas originales. Ios capítulos del libro deben responder $\alpha$ una secuencia lógica conforme a la naturaleza del tema elegido. Es natural que se empiece con un concepto o definición de la materia que se estudia, procurándose escribir frases y pórrafos cortos, sin incisos largos. La precisión es ctra de las virtudes del escritor, evitándose, por lo tanto, las digresiones o vaguedades que no vengan al caso, como así mismo el lenguaje excesivamente conciso que haga difícil captar el pensamiento a quien no estuviese muy familiarizado con el tema. Por eso es prudente el uso constante de un diccionorio que permita utilizar los vocablos adecuadamente.

El rigor lógico no sólo ha de aplicarse al lenguaje, empleando las palabras en su sentido natural y técnico, sino que también a la exposición con el objeto de evitar caer en redundancias a través de ella, pues las repeticiones de ideas o conceptos en el desarrollo del trabajo, rompen la armonía que habrá de caracterizarle y pueden conducir a la confusión del que leyere.

No trate de imitar el estilo de ningún autor, por famoso que éste sea: ni menos aún de plagiar. Lo primero equivaldría a desvirtuar o castrar su personalidad, $Y$, lo segundo, incurriría en el delito más grave que puede cometer el investigador: adulterar la verdad.

Resaltará sus propias ideas y lo dirá llanamente cuando sean ideas ajenas. En este supuesto, hará siempre la cita de la fuente directa o indirecta utilizada. Puede acudir, para ello, que debe ser lo más frecuente, a sintetizar con sus propias palabras el pensamiento del autor o a copiarle literalmente lo que diga, en cuyo caso le encerrará entre comillas. No abuse de las citas, ni las haga muy largas; en el epígrafe anterior le hemos puesto algunos ejemplos. Que no le tengan que llamar autor "entre comillas", porque su libro sea una simple recolección de citas desde el comienzo hasta el fin.

El autor citado se indicará al final de la página, del capítulo o del libro. La cita se indica mediante una llamada, generalmente con un número puesto entre paréntesis, que va del número (1) en adelante, comenzándose de nuevo la numeración o al empezar la nueva página o el nuevo capítulo; o, lo que es más usual, que sea una numeración corrida desde el primer capítulo hasta el final, pues la Presentación o Introducción tendrá su propia numeración.

Para el lector lo más cómodo es que la cita se haga a pie de página, porque con una ojeada puede enseguida abarcar el texto completo, mientras que si va al final del capítulo o de la obra, siempre le resultará engorroso su consulta. El que escribe, no obstante, prefiere hacer una numeración corrida y poner la cita entre poréntesis a fin de que, posteriormente, cuando se escriba a máquina la secretaria conserve la numeración 
entre paréntesis, pero la cita con el msimo número lo transcriba, después de haber escrito todo el trabajo, en cuartillas o pliegos aparte bajo el título de Notas, en las que se repetirá la numeración corrida del texto con la cita de los autores, obra y las referencias que estime oportuno hacer el investigador. Luego es fácil, cuando se lleva el trabajo a la imprenta, que lo compongan, si se desea, con las llamadas a pie de máquina.

De más esła decir, que la cita se utiliza para referirse a algo importante, característico $u$ original expresado por un autor; por lo tanto, no debe hacerse, cuando se trata de conceptos que son patrimonio común; v. gr., el contrato es el acuerdo de voluntades; $2+2=4$.

Ia cita se puede hacer o refiriéndose al autor en el mismo texto de la obra, o recogiendo su nombre en ella. Por lo general, se ha de hacer en el idioma en que se escribe el libro; sólo; como excepción, en lengua extranjera. Esí, por ejemplo, si hablamos del concepto de la justicia, podemos expresar, que Tomás de AQUINO al referirse a ella, establece que "requiert la diversité des sujets, et de lá vient qu'elle n'a lieu que d'un homme ó un outre".

En este caso citado, con mayor razón debiera haberse traducido al español la frase - suponiendo que en este idioma se hiciera la investigación-, puesto que Tomás de AQUINO tompoco la escribió en francés sino en latín, que es en la lengua en que acaso podría escribirse.

Il número de la cita, generalmente, se coloca como aquí lo hemos hecho; esto es, después que expusimos el pensamiento del autor, si bien $\alpha$ veces sueie colocarse después del nombre del autor citado; v. gr., Tomás de AQUINO (1). Esto, claro es, cuando se le cita en el texto.

Lo normal es que el trabajo se escriba a doble espacio a máquina por una sola cara del papel y dejando márgenes suficientes a los lados: de 1/2 pulgadas en el lado izquierdo y arriba y de 1 pulgada en el lado derecho $y$ abajo. Los párrafos deben comenzar 7 espacios del margen izquierdo. Lạs citas en el texto y las notas a pie de página se escribirán a espacio sencillo con márgenes mayores para que se destaquen del contexto del trabajo.

Cuando àespués de hecha la cita de un libro más adelante se le vuelve a citar, intercaladas en el intermedio citas de otros, entonces no hay necesidad de volver a escribir el título siendo suficiente con poner el nombre del outor $y$, seguidamente, op. cit. $u$ ob. cit., que quiere decir: "obra citada"; v. gr., LACHANCE, op cit., p. 400 ó pp. ó págs. 400-401. Empero, eso sí, decididos por la forma de la expresión la utilicemos siempre a lo largo del trabajo, ora sea op. u ab., ora sea p. o pág.

Una advertencia debemos hacer: Los artículos de un cuerpo legal o disposición legislativa y las fechas de los fallos o sentencias de la jurisprudencia se harán en el mismo texto del trabajo y no hay necesidad de hacer llamada para referirse al código que se cita, basta decir del Código ci-

5.-II, II, q 58, a. 2 cit. por LACHANCE; "LE CONCEPT DE DROIT SELON ARLSTOTE ET S. THOMAS, Paris, 1933, p. 329. 
vil panameño en su artículo tal, o el Código civil italiano de 1865, puesto que el vigente en Italia es de fecha posterior.

En la cita, se puede recoger la opinión de varios autores; v. gr., LACHANCE, op. cit., p. 500; LEGAZ y LACAMBRA, op. cit., pp. 10-11; y PEIDRO PASTOR, op. cit., p. 4. También se pueden hacer explicaciones en la cita o transcribir en ella el pensamiento del autor en forma sintetizada o literal. Si en un tema se recomienda la consulta de otras obras, se usan las expresiones ctr. o véase. Así nos escribe Luis LEGAZ y LACAMBRA, en su jibro "Filosofía del Derecho", Barcelona, Ed. Bosch, 1961, ed. 2a., p. 380, que "las constituciones de cada Estado (normas primarias del sistema jurídico estatal) son secundarias por respecto a la constitución de ese sistema jurídico más amplio". (3)

La s.s. significan siguientes.

Si hemos citado un autor y seguidamente volvemos a hacerlo, tratándose de la misma obra, se usa el término lbidem, p. 40 . Esto quiere decir, "en el mismo lugar", pero en distinta página. Si, por el contrario, se trata del mismo autor, obra y página, se emplea Loc. cit., que indica "lugar citado", o idem, que significa "la misma persona", y se refiere a la persona ya mencionada en la nota inmediatamente precedente. También se usa loc cit. en vez de op. cit., en el caso de un artículo en una publicación periódica.

\author{
n.d., quiere decir "sin fecha" \\ supra, equivale a "sobre" \\ intra, significa "abajo" \\ lex cit., quiere decir "ley citada'l. En plural es leges cit.
}

Hay autores que acostumbran a citar los cuerpos legales y las revistas valiéndose de abreviaturas cada vez que las mencionan; v. gr., C.c. por Código Civil: R.D.P. por Revista de Derecho Privado. En tales casos, en la bibliografía se deben incluir estas abreviaturas en el lugar que le corresponda remitiendo al título completo mediante la indicación Véase seguida de Código Civil o Revista de Derecho Privado, sin necesidad de más datos. Los datos bibliográficos completos se harán mención en la notá que se refiere a la cita.

Finalmente, viene la Conclusión que, como sucede, por lo general, con la Introducción, son los dos últimos capítulos redactados. En la Conclusión deben formularse en términos precisos las formulaciones a que llega el investigador en su trabajo, como también aquellos problemas que deja esbozados 0 sin abordar, a fin de que otros estudiantes o estudiosos lo hagan, puesto que el esfuerzo científico es resultado de la colaboración de las generaciones humanas. Por ello es que propugnamos una educación comunitaria o solidarista, por considerar que el hombre se debe a sus semejantes y no a satisfacer su "ego". Afortunadamente hemos quemado la etapa histórica del hombre-individuo o del pequeño dios $y$ nos encamina-

6.-Para una típología de los conceptos de constitución en sentido jurídico-positivo, VEASE SANCHEZ AGESTA, LECCIONES DE DERECHO POLITICO. Granada, 1947, págs. 344 y 33.; M. GARCIA PELAYO, DERECHO CONSTITUCIONAL COMPARADO, págs. 32 y s.s. 
mos, resueltamente, hacia la concepción del hombre-persona, que le concibe como puesto siempre al servicio del bien de la comunidad, sin que para ello tenga que despojarse de sus atributos personales inalienables o trascendentes.

Se cierra el trabajo de investigación con las tablas $\circ$ índices, que comprenden la bibliogrofía citada, refiriéndose, por su orden, a las obras generales, a las monografías sobre el tema, los artículos de Revistas, (en todas ellas, ordenadas las obras, por orden alfabético de los apellidos de los autores), los Textos legales y jurisprudenciales (siguiendo el orden de fechas de menor a mayor). También se debe acompañar, ademós del indice general con sus partes, títulos, capítulos y epígrafes, los índices de cutores y de materiass, recogiéndose, en este último, los conceptos principales tratados en la obra.

\section{Palabras finales.}

Al llegar aquí, caro estudiante o investigador, te sentirós al principio entusiasmado con el alumbramiento de tu primer o nuevo hijo intelectual. Siempre el amor paterno, ciega; pero después, transcurrido el tiempo, sobre todo si llegas a releer el trabajo en letras de imprenta, comenzarás a apreciar sus defectos, sin por ello dejarlo de querer, puesto que por algo es fruto de tu espíritu creador. Además, todo libro en el camino de una vida, es prueba de la laboriosidad humana y peldaño hacia conquistas de más elevado rango espiritual.

Tu profesor, colega o amigo que esto escribió te lo ofrece sinceramente por si puede ser de utilidad para ti, en el quehacer de la vida universitaria o profesional. Ojalá así sea, por lo menos está seguro de que esta ofrenda va plena de sacrificio, entrega y felicidad a través de una vida humana gallardamente consagrada al estudio, a los estudiosos, $\alpha$ los rebeldes auténticos, a la Universidad y a esa humanidad doliente que lucha por un mundo mós traternal. 